Mannheim Working Paper Series on

Risk Theory, Portfolio Management and Insurance

No. 186

\title{
VaR- and CVaR-minimal Futures Hedging Strategies: An Analytical Approach
}

Peter Albrecht, Markus Huggenberger, Alexandr Pekelis 


\title{
VaR- and CVaR-minimal Futures Hedging Strategies: An Analytical Approach
}

\author{
Peter Albrecht, Markus Huggenberger*, Alexandr Pekelis \\ University of Mannheim, Germany
}

This version: August 10, 2012

First version: August 15, 2011

\begin{abstract}
Although Value at Risk (VaR) and Conditional Value at Risk (CVaR) have been established as standard techniques in many fields of risk management and portfolio selection, the literature rarely applies these risk measures to futures hedging. The purpose of this paper is to characterize analytically VaR- and CVaR-minimal hedging strategies. We apply results about quantile derivatives to obtain first order conditions that hold under weak assumptions on the underlying return distribution. We then focus on conditionally elliptical return processes, which enables us to derive closed form expressions for these conditions. In the case of hedging with a single futures contract, these expressions can explicitly be solved for (C)VaR-minimal hedge ratios. Hedging strategies based on these results account for the risk caused by the fat tails of return distributions. In a further step, we extend our characterizations of optimal hedging strategies to mixtures of elliptical distributions. This generalization allows capturing distributional asymmetries, which was found to be highly important for tail based risk measurement. Overall, our findings can be used to implement (C)VaR-minimal hedging rules for most econometric models employed in the futures hedging literature, including multivariate GARCH and regime switching models.
\end{abstract}

Keywords: Futures Hedging; Quantile Derivatives; Mixture Distributions; Elliptical Distributions; Value at Risk; Conditional Value at Risk

JEL classifications: G11, G32.

\section{Introduction}

Across major asset classes, hedging with futures contracts is a popular tool for managing and reducing the price risk of a portfolio of fixed spot positions. The design of riskminimal hedging strategies relies on two main assumptions: (i) an econometric model for the joint distribution of stock and futures price changes and (ii) an objective function

\footnotetext{
* Corresponding author. Tel. +49621 18116 79. E-mail: huggenberger@bwl.uni-mannheim.de.
} 
that describes the investor's perception of risk. Following the pioneering work of Johnson (1960), most of the literature still uses the variance when quantifying the risk of a hedging strategy, which corresponds to the traditional portfolio selection approach. ${ }^{1}$ This paradigm is opposed by the development in other fields of risk management, where the application of shortfall risk measures increasingly gained significance. In particular, Value at Risk (VaR) and Conditional Value at Risk (CVaR) evolved into standard tools in risk measurement and portfolio optimization. Nevertheless, only few attempts ${ }^{2}$ have been made to develop hedging strategies based on these risk measures. In order to fill this gap, we derive (C)VaR-minimal hedging rules that apply to a very broad range of econometric models.

Analytically, such hedge positions have only been investigated given the assumption that the distributions of spot and futures returns are conditionally normal (Hung et al., 2006) or, alternatively, by using semi-parametric techniques for estimating VaR and CVaR (Cao et al., 2010). Other studies, which use non-parametric VaR-estimates (Harris and Shen, 2006) or more flexible econometric models (Lee and Hung, 2007; Chang, 2010), solely rely on numerical optimization techniques for the determination of optimal strategies.

In this study, we provide analytical characterizations of (C)VaR-minimal hedging strategies for various parametric return models. The setting that we present covers multivariate $^{3}$ as well as dynamic hedging problems. Our contribution to the literature is threefold. First, we apply results on quantile derivatives in order to obtain first order conditions for (C)VaR-minimal hedging positions under weak assumptions on the underlying return distributions. Second, we focus on conditionally elliptical return processes to find closed form expressions of the conditions given above. In the case of single futures hedging, these conditions can be explicitly solved for the optimal hedge ratios. In particular, our results generalize findings for the normal distribution reported by Hung et al. (2006). When VaR or CVaR is used for risk management, this generalization is highly relevant, since these risk measures are particularly sensitive to the fat tails of the underlying loss distributions. In addition, these findings apply to all kinds of GARCH-type return processes with elliptical residuals, which are a widespread econometric assumption in the hedging literature. Third, we extend our framework by incorporating mixtures of elliptical distributions. This allows us to capture asymmetries in return distributions, which was shown to be important when considering tail risk. In addition, our results

$1 \quad$ A large strand of the hedging literature suggests time-varying models for the covariance of spot and futures price changes, which are then used to compute and test minimum-variance hedging rules (Baillie and Myers, 1991; Kroner and Sultan, 1993; Lee, 2010).

2 Besides the studies mentioned below, Cotter and Hanly (2006) and Alizadeh et al. (2008) use these risk measures for the evaluation of hedging performance. An alternative shortfall risk approach can be found in Lien and Tse (1998), who employ lower partial moments as objective function.

3 This term refers to the simultaneous determination of short positions in several futures (Anderson and Danthine, 1980; Gagnon et al., 1998; Eaker and Grant, 1987). 
for mixtures are applicable if returns are assumed to follow regime switching processes, which is a very recent development in the futures hedging literature.

Finally, we propose to build hedging strategies based on demeaned modifications of VaR and CVaR. In contrast to the original risk measures, these modifications do not contain an implicit trade-off between the expected return and a deviation measure of the hedged portfolio. Consequently, the corresponding strategies are directly comparable to the minimum-variance approach and they do not suffer from the poor out-of-sampleperformance that is associated with mean forecasts. ${ }^{4}$

\section{Model Set-Up and General Results}

We analyze a multivariate hedging problem, i.e., we intend to reduce the risk of a portfolio consisting of long positions in $k$ assets by selling short $l$ futures. Let $\left(\boldsymbol{S}_{t}\right)_{t \in \mathbb{N}}=\left(S_{t, 1}, \ldots, S_{t, k}\right)_{t \in \mathbb{N}}^{\prime}$ and $\left(\boldsymbol{F}_{t}\right)_{t \in \mathbb{N}}=\left(F_{t, 1}, \ldots, F_{t, l}\right)_{t \in \mathbb{N}}^{\prime}$ describe the vector valued discrete time processes of the spot and futures prices. Additionally, $\boldsymbol{n}=\left(n_{1}, \ldots, n_{k}\right)^{\prime}$ denotes the unit spot holdings and $\boldsymbol{m}=\left(m_{1}, \ldots, m_{l}\right)^{\prime}$ represents the units of short futures positions.

Neglecting margin payments, the one-period return of the hedged portfolio is

$$
R_{h, t}=\frac{\boldsymbol{n}^{\prime}\left(\boldsymbol{S}_{t}-\boldsymbol{S}_{t-1}\right)-\boldsymbol{m}^{\prime}\left(\boldsymbol{F}_{t}-\boldsymbol{F}_{t-1}\right)}{\boldsymbol{n}^{\prime} \boldsymbol{S}_{t-1}}=\boldsymbol{x}^{\prime} \boldsymbol{R}_{S, t}-\boldsymbol{h}^{\prime} \boldsymbol{R}_{F, t}
$$

where $\boldsymbol{x}=\left(x_{1}, \ldots, x_{k}\right)^{\prime}$ and $\boldsymbol{h}=\left(h_{1}, \ldots, h_{l}\right)^{\prime}$ are the vectors of portfolio weights in the spot and futures positions, respectively. ${ }^{5}$ For $j=1, \ldots, l, \boldsymbol{h}$ is defined as

$$
h_{j}=\frac{m_{j} F_{t-1, j}}{\boldsymbol{n}^{\prime} \boldsymbol{S}_{t-1}}
$$

The random vectors $\boldsymbol{R}_{S, t}$ and $\boldsymbol{R}_{F, t}$ correspond to one-period spot and (formal) futures returns, i.e.,

$$
R_{S_{t, i}}=\frac{S_{t, i}-S_{t-1, i}}{S_{t-1, i}} \quad \text { and } \quad R_{F_{t, j}}=\frac{F_{t, j}-F_{t-1, j}}{F_{t-1, j}}
$$

for $i=1, \ldots, k$ and $j=1, \ldots, l$.

Our analysis is based on the distribution of these return vectors as the price processes themselves are non-stationary. ${ }^{6}$ In particular, we will discuss how to choose optimal

$4 \quad$ These problems are especially documented in the portfolio selection literature (Jagannathan and Ma, 2003; DeMiguel et al., 2009).

$5 \quad$ If $k=l=1$, then obviously $x=1$ and $h=\frac{m_{1} F_{t-1,1}}{n_{1} S_{t-1,1}}$ corresponds to the hedge ratio as, e.g., used in Chen et al. (2003).

6 The literature is contentious about the identification of markets in which spot and futures prices 
amounts of futures to be sold in $t-1$ based on an available information set $\mathcal{F}_{t-1}$. We write $\mathrm{P}^{t-1}(\cdot), \mathbb{E}^{t-1}[\cdot]$ and $Q_{\alpha}^{t-1}(\cdot)$ as compact notation for probabilities, expectations and quantiles conditional on $\mathcal{F}_{t-1}$. If $\mathcal{F}_{t-1}$ does not contain any relevant information, meaning that $\left(\boldsymbol{R}_{S, t}, \boldsymbol{R}_{F, t}\right)$ is independent of $\mathcal{F}_{t-1}$, and the return processes are indeed stationary, then we obtain time-invariant unconditional hedging strategies. In contrast, the assumption that $\mathcal{F}_{t-1}$ contains past observations ${ }^{7}$, together with the application of time series models, leads to time-varying conditional hedging strategies (Cecchetti et al., 1988; Baillie and Myers, 1991; Kroner and Sultan, 1993). An alternative technique is to include explanatory variables into the filtration (Ederington and Salas, 2008). Our approach is compatible with all of these settings. It only relies on the following assumption with respect to the conditional distribution of the return process.

Assumption 1 The distribution of $\left(\boldsymbol{R}_{S, t}, \boldsymbol{R}_{F, t}\right)$ given $\mathcal{F}_{t-1}$ has a strictly positive density and $\boldsymbol{R}_{S, t}$ as well as $\boldsymbol{R}_{F, t}$ have bounded expectations for all $t \in \mathbb{N}$.

In order to circumvent the disadvantages of the variance, our approach is based on downside risk measures. In particular, we focus on the minimization of the $\mathrm{VaR}_{\alpha}$ and the $\mathrm{CVaR}_{\alpha}$ of the hedged portfolio. Assumption 1 allows for simple definitions of these risk measures. Under this assumption, the $\alpha$-quantile of $R_{h, t}$ is uniquely defined for any confidence level $0<\alpha<1$ by

$$
\mathrm{P}^{t-1}\left(R_{h, t} \leq Q_{\alpha}^{t-1}\left(R_{h, t}\right)\right)=\alpha
$$

Considering negative returns as (relative) losses, we may $\operatorname{set}^{8}$

$$
\operatorname{VaR}_{\alpha}^{t-1}\left(R_{h, t}\right)=Q_{1-\alpha}^{t-1}\left(-R_{h, t}\right)=-Q_{\alpha}^{t-1}\left(R_{h, t}\right)
$$

Next, we define $\mathrm{CVaR}_{\alpha}$ as the average loss in case $R_{h, t}$ falls short of its $\alpha$-quantile (Rockafellar et al., 2006); formally we have

$$
\operatorname{CVaR}_{\alpha}^{t-1}\left(R_{h, t}\right)=\mathbb{E}^{t-1}\left[-R_{h, t} \mid R_{h, t}<Q_{\alpha}^{t-1}\left(R_{h, t}\right)\right]
$$

For absolutely continuous distributions, this simple $\mathrm{CVaR}_{\alpha}$ definition corresponds to various similar tail risk measures found in the literature. ${ }^{9}$ Notably, it coincides with the

are cointegrated (Brenner and Kroner, 1995). However, it is unquestionable that the price processes are integrated.

7 In this case $\mathcal{F}_{t-1}$ corresponds to the natural filtration generated by the return processes, i.e., $\mathcal{F}_{t}=$ $\sigma\left(\boldsymbol{R}_{S, k}, \boldsymbol{R}_{F, k}, k \in \mathbb{N}_{0}, k \leq t\right)$.

8 The superscript underlines that we consider risk measures calculated for the conditional distribution.

9 These include Expected Shortfall (Tasche, 2002) and Tail Conditional Expectation (Acerbi and Tasche, 2002). 
Tail Value-at-Risk (Dhaene et al., 2006),

$$
\operatorname{TVaR}_{\alpha}^{t-1}\left(R_{h, t}\right)=\frac{1}{\alpha} \int_{0}^{\alpha} \operatorname{VaR}_{u}^{t-1}\left(R_{h, t}\right) \lambda(\mathrm{d} u)
$$

which was shown to be a coherent measure of risk in the sense of Artzner et al. (1999). This means that it satisfies the four axioms (i) translation invariance, (ii) subadditivity, (iii) positive homogeneity and (iv) positivity.

In contrast to the variance, which is a deviation measure ${ }^{10}$, both $\mathrm{VaR}_{\alpha}$ and $\mathrm{CVaR}_{\alpha}$ depend on the expected value of the underlying loss random variable. ${ }^{11}$ They hereby contain an implicit trade-off between the mean and some deviation measure. In order to improve the comparability between $(\mathrm{C}) \mathrm{VaR}_{\alpha}$-minimal and minimum variance hedging strategies, we define demeaned modifications of $\mathrm{VaR}_{\alpha}$ and $\mathrm{CVaR}_{\alpha}$ as

$$
\operatorname{MVaR}_{\alpha}^{t-1}\left(R_{h, t}\right):=\operatorname{VaR}_{\alpha}^{t-1}\left(R_{h, t}-\mathbb{E}^{t-1}\left[R_{h, t}\right]\right)
$$

and

$$
\operatorname{MCVaR}_{\alpha}^{t-1}\left(R_{h, t}\right):=\operatorname{CVaR}_{\alpha}^{t-1}\left(R_{h, t}-\mathbb{E}^{t-1}\left[R_{h, t}\right]\right),
$$

respectively. ${ }^{12}$ This immediately implies

$$
\begin{array}{r}
\operatorname{MVaR}_{\alpha}^{t-1}\left(R_{h, t}\right)=\operatorname{VaR}_{\alpha}^{t-1}\left(R_{h, t}\right)+\mathbb{E}^{t-1}\left[R_{h, t}\right] \\
\operatorname{MCVaR}_{\alpha}^{t-1}\left(R_{h, t}\right)=\operatorname{CVaR}_{\alpha}^{t-1}\left(R_{h, t}\right)+\mathbb{E}^{t-1}\left[R_{h, t}\right] .
\end{array}
$$

To simplify notation and to stress the dependence of these risk measures on $\boldsymbol{h}$, we rewrite them as functions of the hedging weights. For example, in case of $\mathrm{VaR}_{\alpha}$, we will use the notation

$$
\operatorname{VaR}_{\alpha}^{t-1}:=\operatorname{VaR}_{\alpha}^{t-1}(\boldsymbol{h}):=\operatorname{VaR}_{\alpha}^{t-1}\left(R_{h, t}(\boldsymbol{h})\right) .
$$

Eventually, we obtain the following optimization problems:

$$
\begin{array}{r}
\boldsymbol{h}_{\mathrm{VaR}}^{*}=\operatorname{argmin}_{\boldsymbol{h} \in \mathbb{R}^{m}} \operatorname{VaR}_{\alpha}^{t-1}(\boldsymbol{h}), \\
\boldsymbol{h}_{\mathrm{MVaR}}^{*}=\operatorname{argmin}_{\boldsymbol{h} \in \mathbb{R}^{m}} \operatorname{MVaR}_{\alpha}^{t-1}(\boldsymbol{h}),
\end{array}
$$

$10 \quad$ See Rockafellar et al. (2006) for a discussion on deviation measures.

11 This fact can be illustrated considering the expressions for $\operatorname{VaR}_{\alpha}^{t-1}$ and $\mathrm{CVaR}_{\alpha}^{t-1}$ in case $R_{h, t} \mid \mathcal{F}_{t-1}$ is normally distributed. We then have $\operatorname{VaR}_{\alpha}^{t-1}=N_{1-\alpha} \sigma\left(R_{h, t} \mid \mathcal{F}_{t-1}\right)-\mathbb{E}^{t-1}\left[R_{h, t}\right]$ and $\mathrm{CVaR}_{\alpha}^{t-1}=$ $\frac{\varphi\left(N_{1-\alpha}\right)}{\alpha} \sigma\left(R_{h, t} \mid \mathcal{F}_{t-1}\right)-\mathbb{E}^{t-1}\left[R_{h, t}\right]$ with $N_{1-\alpha}$ denoting the $(1-\alpha)$-quantile and $\varphi$ the pdf of a standard normal distribution.

$12 \mathrm{MVaR}_{\alpha}$ is, e.g., referred to as Mean-VaR in McNeil et al. (2005). MCVaR $\alpha$ is also known as Shortfall (Bertsimas et al., 2004) or CVaR-Deviation (Rockafellar et al., 2006). 


$$
\begin{array}{r}
\boldsymbol{h}_{\mathrm{CVaR}}^{*}=\operatorname{argmin}_{\boldsymbol{h} \in \mathbb{R}^{m}} \operatorname{CVaR}_{\alpha}^{t-1}(\boldsymbol{h}), \\
\boldsymbol{h}_{\mathrm{MCVaR}}^{*}=\operatorname{argmin}_{\boldsymbol{h} \in \mathbb{R}^{m}} \operatorname{MCVaR}_{\alpha}^{t-1}(\boldsymbol{h}) .
\end{array}
$$

The next theorem provides a general characterization of the solutions to these problems based on first order conditions.

Theorem 1 i) If Assumption 1 holds and the objective functions in (11)-(14) are continuously differentiable in $\boldsymbol{h}$, then the optimal hedge vectors solve

$$
\begin{aligned}
\mathbb{E}^{t-1}\left[\boldsymbol{R}_{F, t} \mid R_{h, t}\left(\boldsymbol{h}_{\mathrm{VaR}}^{*}\right)=-\operatorname{VaR}_{\alpha}^{t-1}\right] & =\mathbf{0}_{m}, \\
\mathbb{E}^{t-1}\left[\boldsymbol{R}_{F, t} \mid R_{h, t}\left(\boldsymbol{h}_{\mathrm{MVaR}}^{*}\right)=-\operatorname{VaR}_{\alpha}^{t-1}\right]-\mathbb{E}^{t-1}\left[\boldsymbol{R}_{F, t}\right] & =\mathbf{0}_{m}, \\
\mathbb{E}^{t-1}\left[\boldsymbol{R}_{F, t} \mid R_{h, t}\left(\boldsymbol{h}_{\mathrm{CVaR}}^{*}\right) \leq-\operatorname{VaR}_{\alpha}^{t-1}\right] & =\mathbf{0}_{m}, \\
\mathbb{E}^{t-1}\left[\boldsymbol{R}_{F, t} \mid R_{h, t}\left(\boldsymbol{h}_{\mathrm{MCVaR}}^{*}\right) \leq-\operatorname{VaR}_{\alpha}^{t-1}\right]-\mathbb{E}^{t-1}\left[\boldsymbol{R}_{F, t}\right] & =\mathbf{0}_{m} .
\end{aligned}
$$

ii) If the risk measure used in the objective function is subadditive and positive homogeneous (given the conditional distribution of $R_{h, t}$ ), then the corresponding optimization problem is convex.

Proof of Theorem 1. The first order conditions in i) are obtained by applying results on quantile derivatives, which were rigorously derived in Hong and Liu (2009); Hong (2009), ${ }^{13}$ to the loss function

$$
L(\boldsymbol{h})=-\boldsymbol{x}^{\prime} \boldsymbol{R}_{S, t}+\boldsymbol{h}^{\prime} \boldsymbol{R}_{F, t}
$$

and by observing that

$$
\frac{\partial \mathbb{E}^{t-1}\left[R_{h, t}\right]}{\partial \boldsymbol{h}}=-\mathbb{E}^{t-1}\left[\boldsymbol{R}_{F, t}\right]
$$

Part ii) follows from the observations that subadditivity and positive homogeneity entail the convexity of a risk measure and that $R_{h, t}$ is linear in $\boldsymbol{h}$.

The convexity result ensures that every local stationary point of the objective function is a global minimum. Therefore, the conditions in the first part of the theorem characterize risk-minimal hedge vectors. From this point of view, $(\mathrm{M}) \mathrm{CVaR}_{\alpha}$-minimal hedging strategies are preferable to $(\mathrm{M}) \mathrm{VaR}_{\alpha}$-minimal strategies due to the afore-mentioned coherence of the $\mathrm{CVaR}_{\alpha}{ }^{14}$ under Assumption 1. Since this is the only material restriction imposed so far, the derived characterizations are rather general. However, at this level of generality, we cannot provide analytical solutions for the conditional expectations

13 An early version of these results can already be found in Gourieroux et al. (2000), Hallerbach (2002) and Bertsimas et al. (2004).

14 Although $\mathrm{MCVaR}_{\alpha}$ is not coherent, it satisfies the conditions in ii). 
in equations (15)-(18). In order to obtain more explicit representations of the optimal hedging weights, in what follows, we focus on specific assumptions regarding the law of the return processes.

\section{Optimal Hedge Vectors for Elliptically Distributed Returns}

In this section, we assume that the conditional distributions of the return vectors are elliptical. As a generalization of the multivariate normal distribution, the family of elliptical distributions exhibits many favorable properties, which are of major importance for financial modeling. First, it contains a multitude of distribution functions, e.g., the tand symmetric stable distributions as well as normal variance mixtures that are able to capture fat tails often observed in financial market return series. Second, it encompasses many of the technical features known from the normal distribution, which often enable explicit computations. For a full account of elliptical distributions, we refer to Fang et al. (1990) or McNeil et al. (2005). In the following, we limit our analysis to a brief exposition.

Definition 1 Let $\boldsymbol{\mu}$ be a real-valued $n \times 1$-vector, $\boldsymbol{\Sigma}$ a symmetric, positive definite $n \times n$ matrix and $\psi$ a real-valued function on $\mathbb{R}$. A $n \times 1$ random vector $\boldsymbol{X}$ is said to follow an elliptical distribution with the parameters $\boldsymbol{\mu}, \boldsymbol{\Sigma}$ and the characteristic generator $\psi$, if its characteristic function is of the form

$$
c_{\boldsymbol{X}}(\boldsymbol{t})=\mathbb{E}\left[\exp \left(i \boldsymbol{t}^{\prime} \boldsymbol{X}\right)\right]=\psi\left(\boldsymbol{t}^{\prime} \boldsymbol{\Sigma} \boldsymbol{t}\right) \exp \left(i \boldsymbol{t}^{\prime} \boldsymbol{\mu}\right)
$$

In this case, we write

$$
\boldsymbol{X} \sim E_{n}(\boldsymbol{\mu}, \boldsymbol{\Sigma}, \psi)
$$

There are several equivalent definitions for elliptical distributions (Owen and Rabinovitch, 1983). In particular, if an elliptical distribution has a density, this density is of the form

$$
f_{\boldsymbol{X}}(\boldsymbol{x})=\operatorname{det}\left(\boldsymbol{\Sigma}^{-1 / 2}\right) g_{n}\left[(\boldsymbol{x}-\boldsymbol{\mu})^{\prime} \boldsymbol{\Sigma}^{-1}(\boldsymbol{x}-\boldsymbol{\mu})\right]
$$

where $g_{n}$ is a scalar function referred to as the density generator. Since the density is a composition of a quadratic form and $g_{n}$, its contours are elliptically shaped, hence the name of the distribution class. Other important distributional properties of $\boldsymbol{X} \sim$ $E_{n}(\boldsymbol{\mu}, \boldsymbol{\Sigma}, \psi)$ are:

(E1) Let $k \in \mathbb{N}, k<n$ and $\boldsymbol{B} \in \mathbb{R}^{k \times n}$ with $\operatorname{rank}(\boldsymbol{B})=k$, then $\boldsymbol{B} \boldsymbol{X} \sim$ $E_{k}\left(\boldsymbol{B} \boldsymbol{\mu}, \boldsymbol{B} \boldsymbol{\Sigma} \boldsymbol{B}^{\prime}, \psi\right)$. 
(E2) If $\boldsymbol{X} \in \mathcal{L}^{2}$ and $c_{\psi}=-2 \psi^{\prime}(0)$, then $\mathbb{E}[\boldsymbol{X}]=\boldsymbol{\mu}$ and $\operatorname{cov}[\boldsymbol{X}]=c_{\psi} \boldsymbol{\Sigma}$.

(E3) Let $\left(\boldsymbol{X}_{1}, \boldsymbol{X}_{2}\right)$ be a partition of $\boldsymbol{X}$. The corresponding partitions of $\boldsymbol{\mu}$ and $\boldsymbol{\Sigma}$ are denoted by $\boldsymbol{\mu}_{1}, \boldsymbol{\mu}_{2}$ and $\boldsymbol{\Sigma}_{11}, \boldsymbol{\Sigma}_{12}, \boldsymbol{\Sigma}_{21}$. If the conditional expectation of $\boldsymbol{X}_{1}$ given $\boldsymbol{X}_{2}=\boldsymbol{x}_{2}$ exists, then

$$
\mathbb{E}\left[\boldsymbol{X}_{1} \mid \boldsymbol{X}_{2}=\boldsymbol{x}_{2}\right]=\boldsymbol{\mu}_{1}+\boldsymbol{\Sigma}_{12} \boldsymbol{\Sigma}_{22}^{-1}\left(\boldsymbol{x}_{2}-\boldsymbol{\mu}_{2}\right)
$$

(E4) If $n=1$, i.e., $X \sim E_{1}\left(\mu, \sigma^{2}, \psi\right)$, then

$$
\begin{aligned}
\operatorname{VaR}_{\alpha}(X) & =Z_{1-\alpha}(\psi) \sigma-\mu, \\
\operatorname{CVaR}_{\alpha}(X) & =\lambda_{1-\alpha}(\psi) \sigma-\mu,
\end{aligned}
$$

where the constants $Z_{1-\alpha}, \lambda_{1-\alpha}$ are independent of $\mu$ and $\sigma$. For the normal and the t-distribution, a sample of theses constants is presented in Table 1.

Table 1: $Z_{1-\alpha}$ and $\lambda_{1-\alpha}$ for the normal and the t-distribution

\begin{tabular}{rrrrr} 
& Normal & t, $\nu=5$ & t, $\nu=4$ & t, $\nu=3$ \\
\hline \hline$Z_{0.90}$ & 1.282 & 1.476 & 1.533 & 1.638 \\
$Z_{0.95}$ & 1.645 & 2.015 & 2.132 & 2.353 \\
$Z_{0.99}$ & 2.326 & 3.365 & 3.747 & 4.541 \\
\hline$\lambda_{0.90}$ & 1.755 & 2.302 & 2.499 & 2.911 \\
$\lambda_{0.95}$ & 2.063 & 2.890 & 3.203 & 3.874 \\
$\lambda_{0.99}$ & 2.665 & 4.452 & 5.221 & 7.003 \\
\hline \hline
\end{tabular}

Note. $\nu$ corresponds to the degrees of freedom parameter of the t-distribution.

See, for example, Fang et al. (1990) for (E1)-(E3) and Landsman and Valdez (2003) for (E4). Using these properties, we are able to derive concrete expressions for the first order conditions given in Theorem $1 .^{15}$

\section{Theorem 2 If}

$$
\left(\begin{array}{c}
\boldsymbol{R}_{S, t} \\
\boldsymbol{R}_{F, t}
\end{array}\right) \mid \mathcal{F}_{t-1} \sim E_{k+l}\left(\left(\begin{array}{c}
\boldsymbol{\mu}_{S, t} \\
\boldsymbol{\mu}_{F, t}
\end{array}\right),\left(\begin{array}{cc}
\boldsymbol{\Sigma}_{S, t} & \boldsymbol{\Sigma}_{S F, t} \\
\boldsymbol{\Sigma}_{S F, t}^{\prime} & \boldsymbol{\Sigma}_{F, t}
\end{array}\right), \psi\right)
$$

for all $t \in \mathbb{N}$ and the requirements of Theorem 1 are satisfied, then:

$\overline{15}$ Note that in this simple case, explicit representations for $\operatorname{VaR}_{\alpha, t \mid t-1}(\boldsymbol{h})$ and $\mathrm{CVaR}_{\alpha, t \mid t-1}(\boldsymbol{h})$ are available and thus optimal hedge ratios could also be derived without using Theorem 1 . However, we prefer this argument because it provides the foundation for the proof of Theorem 3 . 
i) the $\mathrm{VaR}_{\alpha}$-minimal hedge vector $\boldsymbol{h}_{\mathrm{VaR}}^{*}$ solves

$$
\boldsymbol{\mu}_{F, t}-\frac{\boldsymbol{\Sigma}_{F h, t}}{\sigma_{h, t}} Z_{1-\alpha}(\psi)=\mathbf{0}_{m}
$$

and the $\mathrm{CVaR}_{\alpha}$-minimal hedge vector $\boldsymbol{h}_{\mathrm{CVaR}}^{*}$ satisfies

$$
\boldsymbol{\mu}_{F, t}-\frac{\boldsymbol{\Sigma}_{F h, t}}{\sigma_{h, t}} \lambda_{1-\alpha}(\psi)=\mathbf{0}_{m}
$$

where

$$
\begin{aligned}
\boldsymbol{\Sigma}_{F h, t} & =\boldsymbol{\Sigma}_{S F, t}^{\prime} \boldsymbol{x}-\boldsymbol{\Sigma}_{F, t} \boldsymbol{h}, \\
\sigma_{h, t}^{2} & =\boldsymbol{x}^{\prime} \boldsymbol{\Sigma}_{S, t} \boldsymbol{x}-2 \boldsymbol{x}^{\prime} \boldsymbol{\Sigma}_{S F, t} \boldsymbol{h}+\boldsymbol{h}^{\prime} \boldsymbol{\Sigma}_{F, t} \boldsymbol{h} .
\end{aligned}
$$

ii) $\boldsymbol{h}_{\mathrm{MVaR}}^{*}$ and $\boldsymbol{h}_{\mathrm{MCVaR}}^{*}$ correspond to the minimum variance hedge vector $\boldsymbol{h}^{*}$, which is

$$
\boldsymbol{h}_{\mathrm{MVaR}}^{*}=\boldsymbol{h}_{\mathrm{MCVaR}}^{*}=\boldsymbol{h}^{*}=\boldsymbol{\Sigma}_{F, t}^{-1} \boldsymbol{\Sigma}_{S F, t}^{\prime} \boldsymbol{x} .
$$

iii) All optimization problems are convex.

Proof of Theorem 2. See the appendix for a proof of part i). Property ii) is a well known result for elliptical distributions. It can be reproduced by setting $\boldsymbol{\mu}_{F, t}=\mathbf{0}_{m}$ in (28) or (29) and solving for the hedge vector. iii) follows from the coherence of $\operatorname{VaR}_{\alpha}$ and $\mathrm{CVaR}_{\alpha}$ for the case of elliptically distributed loss variables.

It is worth noting that if the futures price vector follows a pure martingale process, i.e., $\mathbb{E}^{t-1}\left[\boldsymbol{F}_{t}\right]=\boldsymbol{F}_{t-1}$, then $\boldsymbol{\mu}_{F, t}=\mathbf{0}_{m}$. In this case, $\mathrm{VaR}_{\alpha^{-}}$and $\mathrm{CVaR}_{\alpha^{-}}$minimal hedge vectors correspond to the minimum variance hedging rule. These results can easily be explained by considering (E2) and (E4). These properties show that after adjusting for the mean of $R_{h, t}, \mathrm{VaR}_{\alpha}$ and $\mathrm{CVaR}_{\alpha}$ are just multiples of the standard deviation.

In the univariate case, it is possible to solve (28) and (29) explicitly for the optimal hedge ratios. Straight forward calculations ${ }^{16}$ lead to the following corollary.

Corollary 1 If $k=l=1$ and under the assumptions of Theorem 2 with

$$
\left(\begin{array}{l}
R_{S, t} \\
R_{F, t}
\end{array}\right) \mid \mathcal{F}_{t-1} \sim E_{2}\left(\left(\begin{array}{c}
\mu_{S, t} \\
\mu_{F, t}
\end{array}\right),\left(\begin{array}{cc}
\sigma_{S, t}^{2} & \rho_{t} \sigma_{S, t} \sigma_{F, t} \\
\rho_{t} \sigma_{S, t} \sigma_{F, t} & \sigma_{F, t}^{2}
\end{array}\right), \psi\right),
$$

$16 \quad$ These calculations involve solving a quadratic equation. Nevertheless, the hedge ratios given in this corollary are unique because only one solution of the quadratic equation solves the original problems in $(28)$ and (29). 
for $t \in \mathbb{N}$, then the optimal hedge ratios are

$$
\begin{gathered}
\boldsymbol{h}_{\mathrm{VaR}}^{*}=\frac{\sigma_{S, t}}{\sigma_{F, t}}\left(\rho_{t}-\mu_{F, t} \sqrt{\frac{1-\rho_{t}^{2}}{Z_{1-\alpha}^{2}(\psi) \sigma_{F, t}^{2}-\mu_{F, t}^{2}}}\right), \\
\boldsymbol{h}_{\mathrm{CVaR}}^{*}=\frac{\sigma_{S, t}}{\sigma_{F, t}}\left(\rho_{t}-\mu_{F, t} \sqrt{\frac{1-\rho_{t}^{2}}{\lambda_{1-\alpha}^{2}(\psi) \sigma_{F, t}^{2}-\mu_{F, t}^{2}}}\right) .
\end{gathered}
$$

These results generalize the $\mathrm{VaR}_{\alpha}$-minimal hedge ratio obtained by Hung et al. (2006) for the case of normal distributions. Note that these findings apply to the majority of econometric models that have been proposed for the estimation of dynamic hedge ratios because most of them combine multivariate GARCH-type covariance models with elliptical residual distributions. ${ }^{17}$

\section{Mixtures of Elliptical Distributions}

Despite the broad applicability of the results obtained so far, there are two important issues that prompt a more generalized characterization of $\mathrm{VaR}_{\alpha^{-}}$and $\mathrm{CVaR}_{\alpha^{-}}$-minimal hedge vectors: First, regime switching models, which are a very recent econometric development in the hedging literature, are not covered by the given framework. Second, although capturing excess kurtosis, elliptical distributions do not account for skewness effects, which were shown to be of major importance for quantile based risk measurement (Kuester et al., 2006).

In order to account for distributional asymmetries, we consider a mixture of elliptical return processes. We assume that the stochastic behavior of spot and futures returns is governed by $r$ different market states. In particular, this section is based on the following assumption:

Assumption $2\left(X_{t}\right)_{t \in \mathbb{N}}$ is a discrete random process with values in $\{1, \ldots, r\}$. The conditional distributions of $\left(\boldsymbol{R}_{S, t}, \boldsymbol{R}_{F, t}\right)$ given $\mathcal{F}_{t-1}$ and $X_{t}$ are elliptical, formally

$$
\left(\begin{array}{l}
\boldsymbol{R}_{S, t} \\
\boldsymbol{R}_{F, t}
\end{array}\right) \mid X_{t}=i, \mathcal{F}_{t-1} \sim E_{k+l}\left(\left(\begin{array}{c}
\boldsymbol{\mu}_{S, t, i} \\
\boldsymbol{\mu}_{F, t, i}
\end{array}\right),\left(\begin{array}{cc}
\boldsymbol{\Sigma}_{S, t, i} & \boldsymbol{\Sigma}_{S F, t, i} \\
\boldsymbol{\Sigma}_{S F, t, i}^{\prime} & \boldsymbol{\Sigma}_{F, t, i}
\end{array}\right), \psi\right)
$$

for $i=1, \ldots, r$ and all $t \in \mathbb{N}$.

The realizations of $\left(X_{t}\right)_{t \in \mathbb{N}}$, which describe the state of the spot and futures markets in $t$, are usually assumed to be unobservable, i.e., they are not included in $\mathcal{F}_{t-1}$. For our hedging analysis, we need predictive state probabilities ${ }^{18}$, meaning the probabilities

17 See, for instance, Baillie and Myers (1991) or Brooks et al. (2002).

18 If $\left(X_{t}\right)_{t \in \mathbb{N}}$ cannot be observed, filtering techniques are used to infer these probabilities. 
of $X_{t}$ given the information in $\mathcal{F}_{t-1}$. These probabilities will be denoted by $\pi_{i}^{t \mid t-1}:=$ $\mathrm{P}^{t-1}\left(X_{t}=i\right)$ for $i=1, \ldots, r$. Under the Assumptions 1 and 2 , we can write the state independent conditional density of spot and futures returns as

$$
f_{\boldsymbol{R}_{S, t}, \boldsymbol{R}_{F, t} \mid \mathcal{F}_{t-1}}\left(\boldsymbol{r}_{S}, \boldsymbol{r}_{F}\right)=\sum_{i=1}^{r} \pi_{i}^{t \mid t-1} f_{\boldsymbol{R}_{S, t}, \boldsymbol{R}_{F, t} \mid X_{t}=i, \mathcal{F}_{t-1}}\left(\boldsymbol{r}_{S}, \boldsymbol{r}_{F}\right)
$$

where $f_{\boldsymbol{R}_{S, t}, \boldsymbol{R}_{F, t} \mid X_{t}=i, \mathcal{F}_{t-1}}, i=1, \ldots, r$, are the densities of $\left(\boldsymbol{R}_{S, t}, \boldsymbol{R}_{F, t}\right)$ conditional on $X_{t}=i$ and $\mathcal{F}_{t-1}$. These densities are often referred to as component densities and $f_{\boldsymbol{R}_{S, t}, \boldsymbol{R}_{F, t} \mid \mathcal{F}_{t-1}}$ is a mixture of these components with mixing weights $\pi_{i}^{t \mid t-1}$. (E1) implies that the joint distribution of $\boldsymbol{R}_{F, t}$ and $R_{h, t}$ conditional on $\mathcal{F}_{t-1}$ and $X_{t}=i$ is also elliptical with

$$
\left(\begin{array}{c}
\boldsymbol{R}_{F, t} \\
R_{h, t}
\end{array}\right) \mid X_{t}=i, \mathcal{F}_{t-1} \sim E_{l+1}\left(\left(\begin{array}{c}
\boldsymbol{\mu}_{F, t, i} \\
\mu_{h, t, i}
\end{array}\right),\left(\begin{array}{cc}
\boldsymbol{\Sigma}_{F, t, i} & \boldsymbol{\Sigma}_{F h, t, i} \\
\boldsymbol{\Sigma}_{F h, t, i}^{\prime} & \sigma_{h, t, i}^{2}
\end{array}\right), \psi\right),
$$

where $\mu_{h, t, i}=\boldsymbol{x}^{\prime} \boldsymbol{\mu}_{S, t, i}-\boldsymbol{h}^{\prime} \boldsymbol{\mu}_{F, t, i}$ for $i=1, \ldots, r$. The parameters $\boldsymbol{\Sigma}_{F h, t, i}$ and $\sigma_{h, t, i}^{2}$ are calculated as in (30) and (31). Writing

$$
f_{h, t \mid i}:=f_{R_{h, t} \mid X_{t}=i, \mathcal{F}_{t-1}} \quad \text { and } \quad F_{h, t \mid i}:=F_{R_{h, t} \mid X_{t}=i, \mathcal{F}_{t-1}}
$$

for the component pdfs and cdfs of $R_{h, t}$, we obtain the following distribution functions for $R_{h, t} \mid \mathcal{F}_{t-1}$

$$
\begin{aligned}
f_{h, t}(r) & :=f_{R_{h, t} \mid \mathcal{F}_{t-1}}(r)=\sum_{i=1}^{r} \pi_{i}^{t \mid t-1} f_{h, t \mid i}(r), \\
F_{h, t}(r) & :=F_{R_{h, t} \mid \mathcal{F}_{t-1}}(r)=\sum_{i=1}^{r} \pi_{i}^{t \mid t-1} F_{h, t \mid i}(r) .
\end{aligned}
$$

Therefore, the quantile risk measures under consideration are given by

$$
\begin{aligned}
\alpha & =\sum_{i=1}^{r} \pi_{i}^{t \mid t-1} F_{h, t \mid i}\left(-\operatorname{VaR}_{\alpha}^{t-1}\right), \\
\mathrm{CVaR}_{\alpha}^{t-1} & =-\frac{1}{\alpha} \sum_{i=1}^{r} \pi_{i}^{t \mid t-1} \mathbb{E}^{t-1}\left[R_{h, t} \mathbf{1}\left(R_{h, t} \leq-\operatorname{VaR}_{\alpha}^{t-1}\right) \mid X_{t}=i\right] .
\end{aligned}
$$

The analytic form of the expectations in (43) depends on the chosen elliptical distribution. ${ }^{19}$ Since the $\operatorname{VaR}_{\alpha}$ has no explicit representation in this case, the derivation of the first order conditions that characterize $\mathrm{VaR}_{\alpha^{-}}$and $\mathrm{CVaR}_{\alpha^{-}}$minimal hedging vectors is not straightforward. In the following theorem, we provide such conditions based on the abstract characterization presented in Theorem 1 .

19 See, e.g., Winkler et al. (1972) for the case of the normal distribution. 
Theorem 3 If Assumption 2 holds and the requirements of Theorem 1 are satisfied, then the $\mathrm{VaR}_{\alpha}$-minimal hedging strategy $\boldsymbol{h}_{\mathrm{VaR}}^{*}$ solves

$$
\sum_{i=1}^{r} \frac{\pi_{i}^{t \mid t-1} f_{h, t \mid i}\left(-\mathrm{VaR}_{\alpha}^{t-1}\right)}{f_{h, t}\left(-\mathrm{VaR}_{\alpha}^{t-1}\right)}\left[\boldsymbol{\mu}_{F, t, i}+\frac{\boldsymbol{\Sigma}_{F h, t, i}}{\sigma_{h, t, i}^{2}}\left(-\mathrm{VaR}_{\alpha}^{t-1}-\mu_{h, t, i}\right)\right]=\mathbf{0}_{m}
$$

Under the same conditions, the $\mathrm{CVaR}_{\alpha}$-minimal hedging strategy $\boldsymbol{h}_{\mathrm{CVaR}}^{*}$ solves

$$
\sum_{i=1}^{r} \frac{\pi_{i}^{t \mid t-1} F_{h, t \mid i}\left(-\mathrm{VaR}_{\alpha}^{t-1}\right)}{\alpha}\left[\boldsymbol{\mu}_{F, t, i}+\frac{\boldsymbol{\Sigma}_{F h, t, i}}{\sigma_{h, t, i}} \mathbb{E}\left[Z \mid Z \leq z_{i}\right]\right]=\mathbf{0}_{m}
$$

where

$$
z_{i}=\frac{-\mathrm{VaR}_{\alpha}^{t-1}-\mu_{h, t, i}}{\sigma_{h, t, i}}
$$

and $Z$ has the spherical distribution generated by $\psi$. The corresponding first order conditions for $\mathrm{MVaR}_{\alpha}$ - and $\mathrm{MCVaR}_{\alpha}$-minimal hedging rules are obtained by subtracting

$$
\boldsymbol{\mu}_{F, t}=\sum_{i=1}^{r} \pi_{i}^{t \mid t-1} \boldsymbol{\mu}_{F, t, i}
$$

from (44) and (45).

See the appendix for a proof of Theorem 3 .

Remark $1 \mathrm{VaR}_{\alpha}$ - and $\mathrm{MVaR}_{\alpha}$-minimal hedging strategies do possibly not exist or are not unique because the corresponding optimization problems are not necessarily convex for mean-variance-mixtures of elliptical distributions.

Remark 2 If the component distributions are Gaussian, $\mathbb{E}\left[Z \mid Z \leq z_{i}\right]=\frac{\varphi\left(z_{i}\right)}{\Phi\left(z_{i}\right)}$ and (45) corresponds to

$$
\frac{1}{\alpha} \sum_{i=1}^{r} \pi_{i}^{t \mid t-1}\left[\boldsymbol{\mu}_{F, t, i} \Phi\left(z_{i}\right)-\frac{\boldsymbol{\Sigma}_{F h, t, i}}{\sigma_{h, t, i}} \varphi\left(z_{i}\right)\right]=\mathbf{0}_{m},
$$

where $\varphi$ and $\Phi$ are the pdf and the cdf of the standard normal distribution.

Eventually, we give three examples for prominent econometric specifications of the process $\left(X_{t}\right)_{t \in \mathbb{N}_{0}}$, for which this theorem can be applied.

Example 1 (Regime Switching Models) The assumption that $\left(X_{t}\right)_{t \in \mathbb{N}_{0}}$ follows a homogeneous (first order) Markov process leads to so called regime switching models, which were introduced by Hamilton (1989). Recent applications of this econometric specification to futures hedging include, for instance, Alizadeh et al. (2008) or Lee (2010). 
Example 2 (Mixture Models) A less complex but still very flexible model structure is obtained by assuming that the state process is an i.i.d. sequence. This implies that there is no time variation in the state probabilities, i.e. $\pi_{i}^{t \mid t-1} \equiv \pi_{i}$ for $i=1, \ldots, r$. This approach, e.g., corresponds to the model in Alexander and Lazar (2006).

Example 3 (Hidden Markov Models) If we restrict the structure given in Example 1, by assuming that the time dependence of the process is solely introduced by the state probabilities, we obtain a model class that sometimes is referred to as hidden markov models (Cappé et al., 2005). For these models, the random vectors $\boldsymbol{R}_{S, t}$ and $\boldsymbol{R}_{F, t}$ are, conditional on $X_{t}$, independent of $\mathcal{F}_{t-1}$.

\section{Conclusion}

In this paper, we studied optimal futures hedging decisions in a discrete time framework for investors whose risk measurement is based on $\mathrm{VaR}_{\alpha}$ or $\mathrm{CVaR}_{\alpha}$. By adopting results on quantile derivatives, we obtained first order conditions in terms of conditional expectations that characterize $(\mathrm{C}) \mathrm{VaR}_{\alpha}$-minimal hedging rules for a very broad class of return models with continuous distributions. To establish closed form expressions of these conditions and the corresponding hedging weight vectors, we next considered return processes with (conditionally) elliptical distributions as well as processes, which switch between such distributions. The solutions derived in these steps can be used to implement $(\mathrm{C}) \mathrm{VaR}_{\alpha}$-minimal hedging strategies for the majority of econometric specifications found in the futures hedging literature. The most important exception are models with jump dynamics, in which the requirements for applying the quantile derivatives are violated.

Although we did not systematically assess the benefits of $\mathrm{VaR}_{\alpha}$ and $\mathrm{CVaR}_{\alpha}$, it is worth noting that $\mathrm{CVaR}_{\alpha}$-based hedging seems preferable. First, the coherence of $\mathrm{CVaR}_{\alpha}$ ensures that our first order conditions always uniquely determine risk minimizing strategies. Second, ignoring the tail risk beyond the chosen confidence level, $\mathrm{VaR}_{\alpha}$ is reported to produce perverse effects when used for investment decisions (Basak and Shapiro, 2001). Nevertheless, $\mathrm{VaR}_{\alpha}$-minimal hedging is worth considering because of its practical importance. In addition, recent studies report that cases in which $\mathrm{VaR}_{\alpha}$ is not subadditive are only relevant to a limited extent in practice (Garcia et al., 2007). 


\section{Appendix}

Proof of Theorem 2 i) Setting $\mu_{h, t}=\boldsymbol{x}^{\prime} \boldsymbol{\mu}_{S, t}-\boldsymbol{h}_{t}^{\prime} \boldsymbol{\mu}_{F, t}$ and using (E1) it is easy to show that the joint distribution of $\boldsymbol{R}_{F, t}$ and $R_{h, t}$ is also elliptical with

$$
\left(\begin{array}{c}
\boldsymbol{R}_{F, t} \\
R_{h, t}
\end{array}\right) \mid \mathcal{F}_{t-1} \sim E_{l+1}\left(\left(\begin{array}{c}
\boldsymbol{\mu}_{F, t} \\
\mu_{h, t}
\end{array}\right),\left(\begin{array}{cc}
\boldsymbol{\Sigma}_{F, t} & \boldsymbol{\Sigma}_{F h, t} \\
\boldsymbol{\Sigma}_{F h, t}^{\prime} & \sigma_{h, t}^{2}
\end{array}\right)\right) .
$$

Based on these parameters and (E3), the first order condition in (15) can be rewritten as

$$
\mathbb{E}^{t-1}\left[\boldsymbol{R}_{F, t} \mid R_{h, t}=-\mathrm{VaR}_{\alpha}^{t-1}\right] \stackrel{(24)}{=} \boldsymbol{\mu}_{F, t}+\frac{\boldsymbol{\Sigma}_{F h, t}}{\sigma_{h, t}^{2}}\left(-\mathrm{VaR}_{\alpha}^{t-1}-\mu_{h, t}\right) .
$$

Applying (E4) and some simple algebra yield

$$
\mathbb{E}^{t-1}\left[\boldsymbol{R}_{F, t} \mid R_{h, t}=-\mathrm{VaR}_{\alpha}^{t-1}\right]=\boldsymbol{\mu}_{F, t}-\frac{\boldsymbol{\Sigma}_{F h, t}}{\sigma_{h, t}} Z_{1-\alpha}(\psi) .
$$

Denoting the density of $R_{h, t}$ conditional on $R_{h, t} \leq-\mathrm{VaR}_{\alpha}^{t-1}$ and $\mathcal{F}_{t-1}$ by $f_{R_{h, t} \mid R_{h, t} \leq-\mathrm{VaR}_{\alpha}^{t-1}}$, we can derive a similar result for the $\mathrm{CVaR}_{\alpha}$-minimal hedge vector. First, we observe that

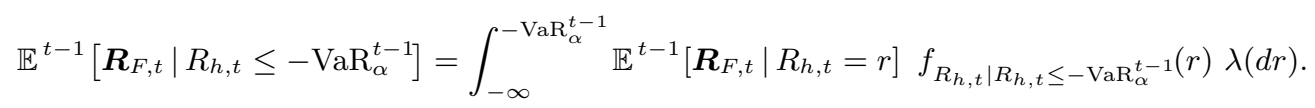

Combining this result with (E3) and using the linearity of the integration operator, it follows that

$$
\begin{aligned}
\mathbb{E}^{t-1}\left[\boldsymbol{R}_{F, t} \mid R_{h, t} \leq-\mathrm{VaR}_{\alpha}^{t-1}\right] & =\boldsymbol{\mu}_{F, t}+\frac{\boldsymbol{\Sigma}_{F h, t}}{\sigma_{h, t}^{2}}\left[\int_{-\infty}^{-\operatorname{VaR}_{\alpha}^{t-1}} r f_{\left.R_{h, t} \mid R_{h, t} \leq-\operatorname{VaR}_{\alpha}^{t-1}(r) \lambda(d r)-\mu_{h, t}\right]}\right. \\
& =\boldsymbol{\mu}_{F, t}+\frac{\boldsymbol{\Sigma}_{F h, t}}{\sigma_{h, t}^{2}}\left[-\mathrm{CVaR}_{\alpha}^{t-1}-\mu_{h, t}\right] .
\end{aligned}
$$

With (E4), we conclude

$$
\mathbb{E}^{t-1}\left[\boldsymbol{R}_{F, t} \mid R_{h, t} \leq-\operatorname{VaR}_{\alpha}^{t-1}\right]=\boldsymbol{\mu}_{F, t}-\frac{\boldsymbol{\Sigma}_{F h, t}}{\sigma_{h, t}} \lambda_{1-\alpha}(\psi) .
$$

Proof of Theorem 3 Noting that

$$
\mathbb{E}^{t-1}\left[\boldsymbol{R}_{F, t} \mid R_{h, t}=y\right]=\frac{\mathbb{E}^{t-1}\left[\boldsymbol{R}_{F, t} \mathbf{1}\left(R_{h, t}=y\right)\right]}{f_{h, t}(y)}
$$

and using the mixture structure of the joint density of $\boldsymbol{R}_{F, t}$ and $R_{h, t}$, we can rewrite conditional expectations like (15) as follows ${ }^{20}$

$$
\begin{aligned}
\mathbb{E}^{t-1}\left[\boldsymbol{R}_{F, t} \mid R_{h, t}=y\right] & =\sum_{i=1}^{r} \frac{\pi_{i}^{t \mid t-1}}{f_{h, t}(y)} \mathbb{E}^{t-1}\left[\boldsymbol{R}_{F, t} \mathbf{1}\left(R_{h, t}=y\right) \mid X_{t}=i\right] \\
& =\sum_{i=1}^{r} \frac{\pi_{i}^{t \mid t-1} f_{h, t \mid i}(y)}{f_{h, t}(y)} \mathbb{E}^{t-1}\left[\boldsymbol{R}_{F, t} \mid R_{h, t}=y, X_{t}=i\right] .
\end{aligned}
$$

20 This decomposition could also be obtained from Bayes' theorem because

$$
\mathrm{P}^{t-1}\left(X_{t}=i \mid R_{h, t}=y\right)=\frac{\mathrm{P}^{t-1}\left(X_{t}=i\right) f_{h, t \mid i}(y)}{\sum_{j=1}^{r} \mathrm{P}^{t-1}\left(X_{t}=j\right) f_{h, t \mid j}(y)}=\frac{\pi_{i}^{t \mid t-1} f_{h, t \mid i}(y)}{f_{h, t}(y)} .
$$


Applying (24) yields

$$
\mathbb{E}^{t-1}\left[\boldsymbol{R}_{F, t} \mid R_{h, t}=y\right]=\sum_{i=1}^{r} \frac{\pi_{i}^{t \mid t-1} f_{h, t \mid i}(y)}{f_{h, t}(y)}\left[\boldsymbol{\mu}_{F, t, i}+\frac{\boldsymbol{\Sigma}_{F h, t, i}}{\sigma_{h, t, i}^{2}}\left(y-\mu_{h, t, i}\right)\right],
$$

which proves (44). For the derivation of the $\mathrm{CVaR}_{\alpha}$-minimal hedging strategy, we conclude by the same reasoning that

$$
\mathbb{E}^{t-1}\left[\boldsymbol{R}_{F, t} \mid R_{h, t} \leq y\right]=\sum_{i=1}^{r} \frac{\pi_{i}^{t \mid t-1} F_{h, t \mid i}(y)}{F_{h, t}(y)} \mathbb{E}^{t-1}\left[\boldsymbol{R}_{F, t} \mid R_{h, t} \leq y, X_{t}=i\right] .
$$

Similar to (53), this can be written as

$$
\begin{aligned}
& \mathbb{E}^{t-1}\left[\boldsymbol{R}_{F, t} \mid R_{h, t} \leq y\right] \\
&=\sum_{i=1}^{r} \frac{\pi_{i}^{t \mid t-1} F_{h, t \mid i}(y)}{F_{h, t}(y)}\left[\boldsymbol{\mu}_{F, t, i}+\frac{\boldsymbol{\Sigma}_{F h, t, i}}{\sigma_{h, t, i}^{2}}\left(\mathbb{E}^{t-1}\left[R_{h, t} \mid R_{h, t} \leq y, X_{t}=i\right]-\mu_{h, t, i}\right)\right] .
\end{aligned}
$$

By the linearity of the conditional expectation, it holds that

$$
\mathbb{E}^{t-1}\left[R_{h, t} \mid R_{h, t} \leq y, X_{t}=i\right]=\mu_{h, t, i}+\sigma_{h, t, i} \mathbb{E}^{t-1}\left[Z \mid Z \leq z_{i}\right],
$$

where $Z$ follows the spherical distribution generated by $\psi$ and

$$
z_{i}=\frac{y-\mu_{h, t, i}}{\sigma_{h, t, i}}
$$

\section{References}

Acerbi, C. and Tasche, D. (2002). On the coherence of Expected Shortfall. The Journal of Banking \&6 Finance, 26(7):1487-1503.

Alexander, C. and Lazar, E. (2006). Normal mixture $\operatorname{GARCH}(1,1)$ : applications to exchange rate modelling. Journal of Applied Econometrics, 21(3):307-336.

Alizadeh, A. H., Nomikos, N., and Pouliasis, P. K. (2008). A Markov regime switching approach for hedging energy commodities. The Journal of Banking \& Finance, 32(9):1970-1983.

Anderson, R. W. and Danthine, J.-P. (1980). Hedging and Joint Production: Theory and Illustrations. The Journal of Finance, 35(2):487-498.

Artzner, P., Delbaen, F., Eber, J.-M., and Heath, D. (1999). Coherent Measures of Risk. Mathematical Finance, 9(3):203-228.

Baillie, R. T. and Myers, R. J. (1991). Bivariate garch estimation of the optimal commodity futures Hedge. Journal of Applied Econometrics, 6(2):109-124.

Basak, S. and Shapiro, A. (2001). Value-at-Risk-Based Risk Management: Optimal Policies and Asset Prices. Review of Financial Studies, 14(2):371-405.

Bertsimas, D., Lauprete, G., and Samarov, A. (2004). Shortfall as a risk measure: properties, optimization and applications. Journal of Economic Dynamics and Control, 28(7):1353-1381.

Brenner, R. J. and Kroner, K. F. (1995). Arbitrage, Cointegration, and Testing the Unbiasedness Hypothesis in Financial Markets. Journal of Financial and Quantitative Analysis, 30(1):23-42.

Brooks, C., Henry, Ó. T., and Persand, G. (2002). The Effect of Asymmetries on Optimal Hedge Ratios. The Journal of Business, 75(2):pp. 333-352.

Cao, Z., Harris, R. D. F., and Shen, J. (2010). Hedging and value at risk: A semi-parametric approach. Journal of Futures Markets, 30(8):780-794. 
Cappé, O., Moulines, É., and Rydén, T. (2005). Inference in hidden Markov models. Springer, New York.

Cecchetti, S. G., Cumby, R. E., and Figlewski, S. (1988). Estimation of the Optimal Futures Hedge. The Review of Economics and Statistics, 70(4):pp. 623-630.

Chang, K.-L. (2010). The optimal value-at-risk hedging strategy under bivariate regime switching ARCH framework. Applied Economics.

Chen, S.-S., Lee, C.-f., and Shrestha, K. (2003). Futures hedge ratios: a review. The Quarterly Review of Economics and Finance, 43(3):433-465.

Cotter, J. and Hanly, J. (2006). Reevaluating hedging performance. Journal of Futures Markets, 26(7):677-702.

DeMiguel, V., Garlappi, L., Nogales, F. J., and Uppal, R. (2009). A Generalized Approach to Portfolio Optimization: Improving Performance by Constraining Portfolio Norms. Management Science, 55(5):798-812.

Dhaene, J., Vanduffel, S., Goovaerts, M., Kaas, R., Tang, Q., and Vyncke, D. (2006). Risk Measures and Comonotonicity: A Review. Stochastic Models, 22(4):573-606.

Eaker, M. R. and Grant, D. M. (1987). Cross-Hedging Foreign Currency Risk. Journal of International Money and Finance, 6:85-105.

Ederington, L. H. and Salas, J. M. (2008). Minimum variance hedging when spot price changes are partially predictable. The Journal of Banking \& Finance, 32(5):654-663.

Fang, K.-T., Kotz, S., and Ng, K. W. (1990). Symmetric multivariate and related distributions. Chapman \& Hall, London.

Gagnon, L., Lypny, G. J., and McCurdy, T. H. (1998). Hedging foreign currency portfolios. Journal of Empirical Finance, 5(3):197-220.

Garcia, R., Renault, E., and Tsafack, G. (2007). Proper Conditioning for Coherent VaR in Portfolio Management. Management Science, 53(3):483-494.

Gourieroux, C., Laurent, J., and Scaillet, O. (2000). Sensitivity analysis of Values at Risk. Journal of Empirical Finance, 7(3-4):225-245.

Hallerbach, W. G. (2002). Decomposing Portfolio Value-at-Risk: A General Analysis. The Journal of Risk, 5(2).

Hamilton, J. D. (1989). A New Approach to the Economic Analysis of Nonstationary Time Series and The Business Cycle. Econometrica, 57(2):357-384.

Harris, R. D. F. and Shen, J. (2006). Hedging and value at risk. Journal of Futures Markets, 26(4):369390.

Hong, L. J. (2009). Estimating Quantile Sensitivities. Operations Research, 57(1):118-130.

Hong, L. J. and Liu, G. (2009). Simulating Sensitivities of Conditional Value at Risk. Management Science, 55(2):281-293.

Hung, J.-C., Chiu, C.-L., and Lee, M.-C. (2006). Hedging with zero-value at risk hedge ratio. Applied Financial Economics, 16(3):259-269.

Jagannathan, R. and Ma, T. (2003). Risk Reduction in Large Portfolios: Why Imposing the Wrong Constraints Helps. The Journal of Finance, 58(4):1651-1683.

Johnson, L. L. (1960). The Theory of Hedging and Speculation in Commodity Futures. The Review of Economic Studies, 27(3):139-151.

Kroner, K. F. and Sultan, J. (1993). Time-Varying Distributions and Dynamic Hedging with Foreign Currency Futures. Journal of Financial and Quantitative Analysis, 28(4):535-551. 
Kuester, K., Mittnik, S., and Paolella, M. S. (2006). Value-at-risk prediction: A comparison of alternative strategies. Journal of Financial Econometrics, 4(1):53.

Landsman, Z. M. and Valdez, E. A. (2003). Tail Conditional Expectations for Elliptical Distributions. North American Actuarial Journal, 7(4):55-71.

Lee, H.-T. (2010). Regime switching correlation hedging. The Journal of Banking ES Finance, $34(11): 2728-2741$.

Lee, M.-C. and Hung, J.-C. (2007). Hedging for multi-period downside risk in the presence of jump dynamics and conditional heteroskedasticity. Applied Economics, 39:2403-2412.

Lien, D. and Tse, Y. K. (1998). Hedging time-varying downside risk. Journal of Futures Markets, 18(6):705-722.

McNeil, A. J., Frey, R., and Embrechts, P. (2005). Quantitative Risk Management: Concepts, Techniques, and Tools. Princeton University Press, Princeton, Oxford.

Owen, J. and Rabinovitch, R. (1983). On the Class of Elliptical Distributions and their Applications to the Theory of Portfolio Choice. The Journal of Finance, 38(3):pp. 745-752.

Rockafellar, R. T., Uryasev, S., and Zabarankin, M. (2006). Generalized deviations in risk analysis. Finance and Stochastics, 10(1):51-74.

Tasche, D. (2002). Expected shortfall and beyond. The Journal of Banking ES Finance, 26(7):1519-1533.

Winkler, R. L., Roodman, G. M., and Britney, R. R. (1972). The Determination of Partial Moments. Management Science, 19(3):290-296. 